\title{
The Effectiveness of an Activity Meter for Overweight Children and Their Caregivers in a Clinical Setting: a Pilot Study
}

\author{
Kaufman $\mathrm{TK}^{*}$, Lynch $\mathrm{BA}^{2}$, Biggs $\mathrm{BK}^{3}$, Quigg $\mathrm{SM}^{4}$, Maxson $\mathrm{JA}^{1}$ and Thacher $\mathrm{TD}^{1}$ \\ ${ }^{1}$ Department of Family Medicine, Mayo Clinic, 200 First Street SW, Rochester, MN 55905, USA \\ ${ }^{2}$ Division of Community Pediatrics and Adolescent Medicine, Department of Pediatric and Adolescent Medicine, \\ Mayo Clinic, 200 First Street SW, Rochester, MN 55905, USA \\ ${ }^{3}$ Division of Child and Adolescent Psychology, Department of Psychiatry and Psychology, Mayo Clinic, 200 First \\ Street SW, Rochester, MN 55905, USA \\ ${ }^{4}$ Division of Primary Care Internal Medicine, Department of Medicine, Mayo Clinic, 200 First Street SW, Rochester, \\ MN 55905, USA
}

${ }^{*}$ Corresponding author: Kaufman TK, MD, Department of Family Medicine, Mayo Clinic, 200 First Street SW, Rochester, MN 55905, USA, Tel: 507-293-8503, E-mail: kaufman.tara@mayo.edu

Citation: Kaufman TK, Lynch BA, Biggs BK, Quigg SM, Maxson JA, et al. (2017) The Effectiveness of an Activity Meter for Overweight Children and Their Caregivers in a Clinical Setting: a Pilot Study. J Obes Overweig 3(1): 102. doi: $10.15744 / 2455-7633.3 .102$

Received Date: January 04, 2017 Accepted Date: May 15, 2017 Published Date: May 17, 2017

\begin{abstract}
Context: Childhood obesity is epidemic in the United States, but low levels of physical activity and high levels of screen time are modifiable risk factors. An activity meter (AM) program, that utilizes an interactive child-focused website and measures the amount and intensity of physical activity, may promote activity and decrease BMI.

Objective: The current study tests the feasibility of using an AM program with child-caregiver pairs in a primary care setting. We hypothesize that participants would rate the system as easy to use, would be motivated with their engagement in physical activity, and would continue to use and recommend to others. We hypothesize the AM system would increase children's physical activity levels and decrease engagement in screen time over the study period. We also measure changes in participants' body mass index (BMI).

Design: Prospective study assessing ease of use and satisfaction with the AM program post-intervention and comparing physical activity and BMI changes in children and caregivers from pre- and post-intervention over a 4-month period.

Setting: A primary care clinic in Rochester, Minnesota.

Participants: Ten children ages 9-14 years with BMI over 85th percentile and their associated caregivers.

Intervention: Zamzee AM and website.

Main Measures: Child and caregiver satisfaction ratings, physical activity measured by AM and survey, screen time engagement measured by survey, and BMI.

Results: Between September 2014 and February 2015, 10 child-caregiver pairs were enrolled, and one pair did not complete the study. Both children and caregivers were satisfied with the usability of the AM and website, were motivated to be more physically active and would continue use of the meter after the study. Six activity meters were lost and five were broken. There were no significant changes in physical activity, screen time, or BMI in either caregivers or children.

Conclusion: This feasibility study suggests promise for use of an AM program in the primary care setting but no effectiveness in improving activity level, screen time or BMI over 4 months. Further study with attention to intervention refinement and larger sample sizes is warranted. Given the prevalence of children with overweight/obesity, innovative ideas to promote physical activity are required.
\end{abstract}

Keywords: Assistive Technology; Childhood Obesity; Children; Exercise; Physical Activity

\section{Introduction}

Obesity in children is a serious health concern in the United States and is on the increase worldwide. In the United States alone, 12.7 million children ages 2-19 years are obese [1]. Studies have shown that obese children are at areater risk than healthy- weight children of becoming obese adults $[2,3]$.

Published guidelines on the prevention and intervention of childhood obesity emphasize lifestyle changes including healthful eating, engagement in regular physical activity, and limited sedentary activities such as screen time [4]. Specific to physical activity, 
the Centers of Disease Prevention and Control recommends children engage in one hour or more of moderate to vigorous physical activity (MVPA) per day [5]; however, estimates suggest only one-quarter of United States youth aged 12-15 years engage in MVPA for at least 60-minutes daily [6]. MVPA is defined as 3-6 metabolic equivalents. Since the primary care setting is the home for most children's medical care, tools that are easy to use and effective at promoting health behavior change through the primary care setting are needed.

Young children tend to spend daily activity in light-intensity. A study by Collings et al found that children who spent time in high light-intensity physical activity had healthy body composition (decrease in sum of skinfolds). They also concluded replacing sedentary time with either light or intense MVPA caused a decrease in skinfolds [7].

A recent study by Barkin et al found a positive association of increasing MVPA in children when parents spent up to 40 minutes per day in MVPA. If the parents went beyond 40 minutes there was a decrease in MVPA for children. This was thought to be due to a parent spending time doing a physical activity that a child could not sustain such as long distance running [8]. Nevertheless it seems to show a trend of children modeling their parents healthy behaviors.

Pedometers have been long established as effective in promoting physical activity among adults, and there is evidence that supports their utility with increasing physical activity levels with children [9], with at least one study indicating greater goalattainment with electronic self-monitoring over paper-and-pencil methods [10]. Pedometer use hasn't been found to improve BMI [9]. Recent growth in the development of e-Health (supported by electronic processes and communication) and $\mathrm{m}$-Health (supported by mobile devices) technologies suggest that effective tools to promote physical activity and other healthy behaviors could be developed [11-13]. When considering that they can be designed for use independently or with limited provider contact, the e- and m-Health technologies hold promise for meeting the needs of a busy primary care practice. Newer technology includes the use of Internet-based intervention platforms, mobile phone applications, messaging systems, and games [11-15]. Drawing from research on effective pediatric obesity interventions, tools that include self-monitoring, goal-setting, and reinforcement of health behaviors are particularly promising [16].

Screen time is another modifiable risk factor for obesity. It is recommended that children have no more than two hours of screen time (computer and watching TV) daily. An excessive amount of screen time can lead to elevated blood pressure, elevated cholesterol and weight gain [17].

The Zamzee Program (http://www.zamzee.com) includes an activity meter (AM) linked to a motivational website designed for children ages 8-14 years. The AM measures the duration and the intensity of activity. Data from the meter can be uploaded to a child-friendly, child-safe website where children and their caregivers can see their activity, choose to share positive messages with other children using the AM, complete physical activity challenges and earn healthy prizes. A Leader Dashboard allows interventionists (e.g. health care providers, teachers) to review activity data for children whose caregivers have allowed the interventionists access.

A study of the Zamzee AM program with 182 middle school children (ages 12-14) by Guthrie et al showed an increase in MVPA that was sustained in those who utilized the AM tool over a six-week period as compared with a control group of children who did not [18]. Another study by Lynch et al. with $5^{\text {th }}$ grade children looked to find if the AM increased physical activity and lowered BMI over an 8 week period. The 7/11 participants did not show decrease in BMI but did show small increase in physical activity (average 17 minutes) [19]. Our review of the literature revealed no reports of clinical AM use with child-caregiver pairs or in the primary care settings. Since children are inspired by their caregivers' behavior it was thought to use a caregiver as a variable in this study.

Our aims were to: 1) Determine the feasibility of the AM system for use with overweight children and their caregivers in the outpatient clinical setting. 2) Determine whether overweight children and caregivers find the AM system to motivate their physical activity and their satisfaction with the AM system. 3) Evaluate the effect of the AM system on the children's physical activity level and recreational screen time and on the BMI in children and their caregivers.

We hypothesize that children and their caregivers would rate the AM system easy to use, describe it as motivating their engagement in physical activity, and indicate satisfaction with using the AM system. Further, we hypothesize that the children's engagement in physical activity would increase over the four-month study period. Considering that BMI change using pedometers has not been observed [9], we assessed pre- to post-intervention BMI change in the children and caregivers but do not expect significant effects on BMI.

\section{Design and Methods}

We conducted a pilot intervention study with children and their caregivers from a semi-urban primary care clinic (family medicine and pediatrics) in Rochester, MN. Inclusion criteria for children were: 1) ages 9-14 years; 2) BMI greater than the $85^{\text {th }}$ percentile for age and gender; 3) caregiver willing to participate; and, 4) computer with internet access and an active email account. The caregiver was defined as the child's biological parent, legally adoptive parent or legal guardian. Eligible children and their caregivers were 
recruited for the study through a letter invitation. The letter encouraged caregivers to contact the study coordinator who described the study, conducted a phone screen to determine eligibility, and scheduled the initial study appointment. Children were not screened for participation in a weight loss program and no data was collected on whether children were in a weight loss program.

The study was approved by the Mayo Clinic Institutional Review Board13.008650. All caregivers provided signed informed consent, and the children ages 9-12 years provided written assent for participation while those ages 13-14 years signed a written consent along with their caregiver.

\section{Study Instruments}

The Zamzee program includes a three-axis accelerometer-based AM and an associated website designed to encourage users to increase their levels of physical activity. The AM measures both the time and intensity of exercise using Global Positioning System technology, fits into a pocket or clips on clothes, and the data can be uploaded with progress tracked on-line. The Zamzee program also tracked minutes in the zone, which is a measure of light physical activity and includes any movement above 2.3 Metabolic Equivalent of Task [20]. When an individual was considered in the zone they would be earning rewards or prizes as a result of physical activity level and completing challenges. An example of a challenge would be doing a certain exercise or increased movement in a time period set by the program. The AM has been approved for use by children as defined by the Child Online Protection Act [21].

Caregivers completed a demographic survey, designed by study investigators to report their relationship to the child, marital status, education level, employment status, age, and race/ethnicity as well as their child's race/ethnicity, age, sex and primary insurance type (Appendix 1).

Satisfaction surveys asked both children and caregivers questions assessing the usability of the AM, the perceived effect of the AM and website on motivating the participant to become more active, and satisfaction with the AM program (defined by intention to continue using it, willingness to recommend it to others, and willingness to pay the current listed retail price for the AM program). Survey questions were developed by investigators with answers recorded on Likert scales. Additional data recorded related to feasibility included contacts between participants and the study team, number of AMs lost or broken, and frequency (uploads) that participants accessed the AM website.

The Self-Administered Physical Activity Checklist (SAPAC) survey is a validated self-report tool for measurement of physical activity and sedentary activity levels in elementary school age children [22] and has also been successfully used with early adolescents [23]. The instrument documents the time a participant spent doing specific physical activities and screen time over the preceding three days. We used this tool for the children and adult caregivers for consistency.

\section{Study Procedures}

There was rolling enrollment beginning September 2014 with the study concluding February 2015. Following enrollment, both the child's and caregiver's height and weight were measured and the BMI was calculated using the Centers for Disease Control and Prevention website calculator [24]. Each participant completed the pre-study AM satisfaction survey and SAPAC survey. Each participating caregiver also completed a family demographic survey. Both participants were given an AM and a study coordinator assisted participants with set-up of their website accounts. Participants were required to agree to the Zamzee Company terms and conditions. The AM website contained helpful information for participants to learn how to use the AM. The study coordinators answered any initial questions and ensured the participants knew how to use the AM before they left enrollment.

Study participants were given instructions to wear the AM whenever possible during waking hours and upload their meter data via a Universal Serial Bus (USB) port to the website at least weekly for four months. There were no accelerometer specifications for minimal wear time. Participants were advised to contact the study coordinator by phone or email if any technical problems occurred with the AM or the website. If no activity had been uploaded (viewable by study staff) on the website for either the child or the caregiver for two consecutive weeks, study staff contacted the adult caregiver to ensure there were no technical issues. The AMs were replaced if they were lost or broken during the study period.

Four months after enrollment, a second SAPAC survey and satisfaction surveys were completed by caregivers and children. Height and weight were measured for all participants on the same scale with the stadiometer used during enrollment to calculate final BMI. Participants could retain the AM for personal use once their active study participation ended. Participants received parking compensation as needed. Each individual in the child-caregiver pair, after completing the study, received remuneration of $\$ 80$.

\section{Data Collection}

Mayo Clinic research staff had access to the Zamzee Program Leader Dashboard, where data from the AMs could be reviewed. All survey (demographic, child and caregiver satisfaction, SAPAC) and BMI data were recorded using a key code in REDCap [25] and exported to Excel. The program JMP 10.0.0 (SAS Institute Inc, Cary, NC) was used for data analysis. Frequencies of participants' responses to the satisfaction surveys were calculated. The median and interquartile ranges (IQR) were determined 
for: 1) minutes of physical activity as recorded by the AM; 2) SAPAC total activity minutes pre- and post-study; and 3) BMI and BMI percentile pre- and post- study). Mean differences between post-intervention and baseline values and $95 \%$ confidence intervals were calculated to determine if changes were significantly different from zero.

\section{Results}

A total of 10 children and 10 caregivers (all were parents) were enrolled (Table 1). Four children were males and six were females. Of the caregivers, one was male and nine female. The median ages of the children and caregivers were 11 years and 39 years, respectively. Nine caregivers were married and eight families had private insurance. One child-caregiver pair did not complete the study and we were unable to reach them for follow up.

\begin{tabular}{|c|c|c|}
\hline & Child $(\mathbf{n}=\mathbf{1 0})$ & Caregiver $(\mathbf{n}=\mathbf{1 0})$ \\
\hline Demographics & & \\
\hline Median age (IQR) & $11(10,13)$ & $38.5(33.75,42.25)$ \\
\hline Gender, male (N, \%) & $4(40)$ & $1(10)$ \\
\hline Race (N) & & \\
\hline White & 8 & 9 \\
\hline Other & 2 & 1 \\
\hline Insurance status & & \\
\hline $\begin{array}{c}\text { Government/ } \\
\text { uninsured }\end{array}$ & 2 & \\
\hline Private & 8 & \\
\hline Marital status & & \\
\hline Live with partner & & 9 \\
\hline Married & & \\
\hline
\end{tabular}

List of Abbreviations: IQR: Interquartile Range

Table 1: Baseline Participant Characteristics

During the four-month study period, six activity meters were lost, five were broken, and there were 38 instances of no website uploads within a two-week timeframe. Of the 6 activity meters that were lost, 3 participants each lost their meters twice. Of the 5 broken meters, 3 participants broke the meter once ( 2 caregivers and 1 child) and 1 participant (caregiver) broke the meter twice.

With assessing usability by survey, a majority of caregivers (seven) had to remind the children to upload data more than one day per week.

Six out of the nine children felt that the AM motivated them to be active and three felt it did not. Eight caregivers felt the AM motivated their children to be active. All caregivers stated the AM helped with their physical activity levels "a little". Children felt it also helped "a little" with increasing activity in eight of the nine respondents.

In assessing satisfaction with the AM, all of the children and caregivers said either "yes" or "maybe" to recommending use of the AM to another family. Children overall were happy with the AM with six saying "yes" they would recommend the AM to a friend. When asked if they would pay $\$ 29.95$ for the AM, seven caregivers said "no". When caregivers were asked if they would recommend the AM to others, seven demarcated "maybe". When asked how often they felt they would use the AM after the study five caregivers said at least one day per week. Children felt similarly about use of AM after the study with six stating they would use the AM at least one day per week.

Zamzee data (Table 2) indicated the children had a median time of 1,282 minutes of MVPA over four months while the caregivers had 641.5 minutes (Table 2). The children had a median of 83 uploads and caregivers had 78 . The number of uploads was unrelated to the change in BMI or activity in children or caregivers.

The median three-day total activity time gleaned from SAPAC surveys of children was 420 minutes at baseline and was 455 minutes post-intervention with six of the nine children increasing their reported total activity time during the study period. The median three-day total activity time for caregivers was 345 minutes at baseline and 340 minutes post-intervention with four caregivers increasing their reported total activity time during the study period. The change in activity in children was not related to their baseline BMI, age, gender, or change in screen time. The change in the children's activity was inversely related with the change in caregiver activity $(\mathrm{r}=-0.65 ; P<0.001)$, but this relationship was greatly influenced by two outliers. When these outliers were excluded, the relationship was no longer significant.

Median three-day total screen time in children was 300 minutes at baseline and 236 minutes post-intervention; and in caregivers was 720 minutes at baseline and 420 minutes post-intervention. Again, none of these differences were significant. There were six caregivers and two children that had reduced screen time post-intervention. The change in screen time in children was not related 
to their baseline BMI, age, gender or change in caregiver screen time. Of note, the post-intervention self-reported three-day activity time was significantly correlated with the MVPA recorded by the AM among children $(\mathrm{r}=0.69 ; P=0.04)$ but not among adults $(\mathrm{r}=-0.14 ; P=0.72)$, confirming reliability of the SAPAC self-reported data in children.

The BMI data are included in Table 2. No significant change in BMI over a four-month period occurred in children or caregivers even when adjusting for baseline weight, age, gender, recorded MVPA, or change in activity or screen time (data not shown). There was a trend toward successful reduction of BMI in children of caregivers with BMI greater than $38(\mathrm{r}=-0.65 ; P=0.06)$.

\begin{tabular}{|c|c|c|c|c|}
\hline Characteristic & $\begin{array}{c}\text { Baseline, Median } \\
\text { (IQR) }\end{array}$ & $\begin{array}{c}\text { Final, Median } \\
\text { (IQR) }\end{array}$ & MD & 95\% CI \\
\hline BMI $\left(\mathrm{kg} / \mathrm{m}^{2}\right)$ & & & & \\
\hline Child & $26.8(24.0-28.8)$ & $26.9(25.1-29.5)$ & 0.18 & -0.33 to 0.68 \\
\hline Caregiver & $31.8(24.3-38.8)$ & $32.0(24.0-39.1)$ & -0.11 & -1.0 to 0.78 \\
\hline Activity (mins/3 days) & & & & \\
\hline Child & $420(165-580)$ & $455(280-930)$ & -26.1 & -664 to 612 \\
\hline Caregiver & $345(235-773)$ & $340(203-780)$ & -16.6 & -306 to 273 \\
\hline $\begin{array}{c}\text { Screen time (mins/3 } \\
\text { days) }\end{array}$ & & & & \\
\hline Child & $300(113-653)$ & $236(145-750)$ & 95 & -90 to 280 \\
\hline Caregiver & $720(293-1,350)$ & $420(270-1,005)$ & -168 & -439 to 102 \\
\hline Zamzee (AM) data ${ }^{\dagger}$ & & & & \\
\hline Mins in zone & & & & \\
\hline Child & & $2,988(797-4,293)$ & & \\
\hline Caregiver & & $1,918(672-2,993)$ & & \\
\hline MVPA & & & & \\
\hline Child & & $1,283(370-2,004)$ & & \\
\hline Caregiver & & $642(237-1,198)$ & & \\
\hline Total data uploads & & & & \\
\hline Child & & & & \\
\hline Caregiver & & & & \\
\hline
\end{tabular}

List of Abbreviations: AM: Activity Meter; CI: Confidence Interval; IQR: Interquartile Range; MD: Mean Difference;

Mins: Minutes; MVPA: Moderate to Vigorous Physical Activity

${ }^{*}$ Minutes in zone defined by Zamzee as a measure of physical activity

${ }^{\dagger}$ Total over four-month period

Table 2: Changes in Health Habits (SAPAC) and Zamzee Data (Four-month Period)*

\section{Discussion}

Findings from the current feasibility study suggest that an AM program can be used with children and their caregivers in a primary care setting. Consistent with a prior study of this system in the school setting [19], nearly all participants in this primary care-based study completed the study, and the majority of both children and caregivers described the system as moderately usable, and having motivated their engagement in physical activity. There were also mixed results regarding continued use of the AM after the study. It is plausible that there was fatigue with the AM program, rewards and excitement after 4 months which negatively impacted the motivation for continued use after the study. The participants were given the AM to keep after the study completion and were planning on continuing to use it. If they had to purchase this; it is inferred the cost was prohibitive.

We did identify challenges to using this technology including participants' difficulty remembering to wear the AM, charge the AM, plug the device in a USB port, and upload the data to the website. As noted earlier there were some meters that were broken or lost which would impede data collection as well as accuracy. Time periods were noted when participants did not access the website, and lost or broke AMs. The research team logged 59 contacts between caregivers and our team which could be a burden on clinicians if they needed to contact participants frequently in regards to AM use. Addressing such challenges in future work may improve participant engagement and outcomes.

Our study results showing lack of improvement in physical activity and decreasing BMI with AM use were similar to what was seen in the study by Lynch et al. [19]. However the study by Guthrie et al. [18] showed statistically significant increased MVPA in adolescents over a brief 6 week period. The absolute increase in MVPA time in the intervention group in this study was only 5 minutes per day as compared to the control group and participants in the intervention group also received slightly more cash rewards which could have influenced results. 
The AM program did not result in improvements in either self-reported or AM-recorded physical activity levels, in self-reported screen time, or BMI in our pilot study. This study was limited in that it did not differentiate screen time for AM use versus other recreational use.

Despite these caveats, the AM program offers a number of potential advances beyond pedometers, which have been demonstrated to increase physical activity, although changes in BMI have not been observed [9]. Compared to a traditional pedometer, which provides feedback on number of steps accrued, the AM program includes feedback regarding duration, intensity, and timing of physical activity; prompts and presents challenges to promote physical activity; and reinforces engagement in physical activity. Inclusion of an interface for caregivers and leaders provides opportunities for adult support that could be explored in future studies. Previous research has demonstrated that child outcomes are related to the engagement of caregivers and caregivers' health behaviors [26,27], which suggests that increasing caregiver support of children's AM program use could improve outcomes. One drawback of the current program is the need to log into the site to obtain feedback and reinforcement. Future AMs that offer immediate feedback and reinforcement, potentially through mobile devices, could be more effective for improving the activity levels and participant engagement with the instrument [28]. Other ways to improve studying an AM would include having a control group, looking into sedentary time with the AM and a much larger study sample.

There were some limitations in this study. There was lack of control or comparison group since this was a pilot study. We also designed our own satisfaction survey and used the SAPAC survey in adults; neither which were validated.

This feasibility study provided initial support for the usability of an AM program with child-caregiver pairs in the primary care setting. Further study is needed to refine how such technology is best used with children and caregivers to determine its efficacy with larger samples. Perhaps an AM that attaches to wrist or gives immediate feedback would be more useful. The AM program alone is not likely to impact long-term activity levels but it may be effective when incorporated in a physical activity program or a group setting.

\section{Acknowledgments}

The study was supported by the Mayo Clinic Center for Translational Sciences Activities through grant number UL1 TR000135 from the National Center for Advancing Translational Sciences, a component of the NIH. The content is solely the responsibility of the authors and does not necessarily represent the official views of the NIH.

The Zamzee Company organized and provided participant activity data to the study team. Thank you to Amie Jones, MD for supporting this research work.

Study data was collected and managed using the Research Electronic Data Capture tool (REDCap), hosted at Mayo Clinic by the Center for Clinical and Translational Science Activities.

\section{References}

1. Ogden CL, Carroll MD, Kit BK, Flegal KM (2014) Prevalence of childhood and adult obesity in the United States, 2011-2012. JAMA 311: 806-14.

2. Serdula MK, Ivery D, Coates RJ, Freedman DS, Williamson DF, et al. (1993) Do obese children become obese adults? A review of the literature. Prev Med 22: $167-77$.

3. The NS, Suchindran C, North KE, Popkin BM, Gordon-Larsen P (2010) Association of adolescent obesity with risk of severe obesity in adulthood. JAMA 304: 2042-7.

4. Barlow SE, Expert Committee (2007) Expert committee recommendations regarding the prevention, assessment, and treatment of child and adolescent overweight and obesity: Summary report. Pediatrics 120: S164-92.

5. Centers for Disease Prevention and Control (2015) How much physical activity do children need? USA.

6. Fakouri T, Hughes JP, Burt VL, Song MK, Fulton JE, et al. (2014) Physical Activity in U.S. Youth aged 12-15 Years, 2012. NCHS Data Brief No. 141, January 2014, USA.

7. Collings PJ, Brage S, Bingham DD, Costa S, West J, et al. (2017) Physical Activity, Sedentary Time, and Fatness in a Biethnic Sample of Young Children. Med Sci Sports Exerc 10.1249/MSS.0000000000001180.

8. Barkin SL, Lamichhane AP, Banda JA, JaKa MM, Buchowski MS, et al. (2017) Parent's Physical Activity Associated With Preschooler Activity in Underserved Populations. Am J Prev Med 10.1016/j.amepre.2016.11.017.

9. Lubans DR, Morgan PJ, Tudor-Locke C (2009) A systematic review of studies using pedometers to promote physical activity among youth. Prev Med 48: 307-15. 10. Cushing CC, Jensen CD, Steele RG (2011) An evaluation of a personal electronic device to enhance self-monitoring adherence in a pediatric weight management program using a multiple baseline design. J Pediatr Psychol 36: 301-7.

11. Free C, Phillips G, Galli L, Watson L, Felix L, et al. (2013) The effectiveness of mobile-health technology-based health behaviour change or disease management interventions for health care consumers: a systematic review. PLoS Med 10: 10.1371/journal.pmed.1001362.

12. Lau PW, Lau EY, Wong del P, Ransdell L (2011) A systematic review of information and communication technology-based interventions for promoting physical activity behavior change in children and adolescents. J Med Internet Res 13: e48.

13. Laplante C, Peng W (2011) A systematic review of e-health interventions for physical activity: an analysis of study design, intervention characteristics, and outcomes. Telemed J E Health 17: 509-23.

14. Lu AS, Kharrazi H, Gharghabi F, Thompson D (2013) A Systematic Review of Health Videogames on Childhood Obesity Prevention and Intervention. Games Health J 2: 131-41. 
15. Delamater AM, Pulgaron ER, Rarback S, Hernandez J, Carrillo A, et al. (2013) Web-based family intervention for overweight children: a pilot study. Child Obes 9: 57-63.

16. Stewart L, Reilly JJ, Hughes AR (2009) Evidence-based behavioral treatment of obesity in children and adolescents. Child Adolesc Psychiatr Clin N Am 18: 189-98.

17. Herrick KA, Fakhouri T, Carlson SA, Fulton JE (2014) TV watching and computer use in U.S. youth aged 12-15, 2012. NCHS Data Brief NO. 157 July 2014, USA.

18. Guthrie N, Bradlyn A, Thompson SK, Yen S, Haritatos J, et al. (2015) Development of an Accelerometer-Linked Online Intervention System to Promote Physical Activity in Adolescents. Plos One 10: 10.1371/journal.pone.0128639.

19. Lynch BA, Jones A, Biggs BK, Kaufman T, Cristiani V, et al. (2015) Implementing Child-focused Activity Meter Utilization into the Elementary School Classroom Setting Using a Collaborative Community-based Approach. J Community Med Health Educ 5: 10.4172/2161-0711.1000379.

20. Byrne NM, Hills AP, Hunter GR, Weinsier RL, Schutz Y (2005) Metabolic equivalent: one size does not fit all. J Appl Physiol 99: $1112-9$.

21. Commission FT (2013) Children's Online Privacy Protection Rule: Not Just for Kids' Sites, USA.

22. Sallis JF, Strikmiller PK, Harsha DW, Feldman HA, Ehlinger S, et al. (1996) Validation of interviewer-and self-administered physical activity checklists for fifth grade students. Med Sci Sports Exerc 28: 840-51.

23. Myers L, Strikmiller PK, Webber LS, Berenson GS (1996) Physical and sedentary activity in school children grades 5-8: the Bogalusa Heart Study. Med Sci Sports Exerc 28: 852-9.

24. Centers for Disease Prevention and Control (CDSCO) About Child \& Teen BMI 2015, USA.

25. Harris PA, Taylor R, Thielke R, Payne J, Gonzalez N, et al. (2009) Research electronic data capture (REDCap)--a metadata-driven methodology and workflow process for providing translational research informatics support. J Biomed Inform 42: 377-81.

26. Fogelholm M, Nuutinen O, Pasanen M, Myöhänen E, Säätelä T (1999) Parent-child relationship of physical activity patterns and obesity. Int J Obes Relat Metab Disord 23: 1262-8.

27. Schoeppe S, Rebar AL, Short CE, Alley S, Lippevelde WV, et al. (2016) How is adults' screen time behaviour influencing their views on screen time restrictions for children? A cross-sectional study. BMC Public Health BMC Public Health 16: 201.

28. Butcher Z, Fairclough S, Stratton G, Richardson D (2007) The effect of feedback and information on children's pedometer step counts at school. Pediatr Exerc Sci 19: 29-38. 